\title{
Globally Coordinated Distributed Storm Water Management System
}

\author{
Luis Montestruque \\ EmNet LLC \\ $121 \mathrm{~S}$ Niles Ave. \#22 \\ South Bend, IN 46617 \\ Imontest@emnet.net
}

\author{
M.D. Lemmon \\ Department of Electrical Engineering \\ University of Notre Dame \\ 275 Fitzpatrick Hall \\ Notre Dame. IN 46556 \\ lemmon@nd.edu
}

\begin{abstract}
This paper describes a highly-distributed wireless cyber physical system (CPS) that is used to manage storm and waste water collection systems at a metropolitan scale. The system, called CSOnet and previously introduced in [6], currently consist of 120 sensors and 12 actuators installed in the sewers of South Bend, IN a Midwestern city with a population of approximately 100,000 people. CSOnetâĂŹs main objective is to reduce wet weather-induced overflows of untreated sewage into the river by dynamically controlling flows as they traverse the network of sewer pipes, pump stations and other infrastructure.
\end{abstract}

\section{Categories and Subject Descriptors}

C.3 [Special-Purpose and Application-Based Systems] Real-time and embedded systems; G.1.6 [Optimization]: Global optimization; I.2.8 [Problem Solving, Control Methods, and Search]: Control Theory

\section{Keywords}

smart water, combined sewer overflow, stormwater, distributed control

\section{INTRODUCTION}

Combined Sewer Overflows (CSO) are a major environmental challenge for more than 700 cities in the United States [1]. According to the USEPA, CSOs are responsible for the release of more than 850 billion gallons of untreated sewage into rivers, lakes, and oceans [2]. CSOs occur in cities where sanitary and storm water flows share the same water collection infrastructure. During rain events, the total sewage flow is larger than the maximum wastewater treatment plant capacity. To prevent street and basement flooding the water collection system was designed to shed the excess flow by releasing untreated wastewater directly into

Permission to make digital or hard copies of all or part of this work for personal or classroom use is granted without fee provided that copies are not made or distributed for profit or commercial advantage and that copies bear this notice and the full citation on the first page. To copy otherwise, to republish, to post on servers or to redistribute to lists, requires prior specific permission and/or a fee.

CySWater'15, April 13-16, 2015, Seattle, WA, USA

Copyright 2015 ACM 978-1-4503-3485-3/15/04 ...\$15.00

http://dx.doi.org/10.1145/2738935.2738948. neighboring rivers or streams. This event is called a combined sewer overflow or CSO event.

Under the Federal Water Pollution Control Act (also known as Clean Water Act) of 1972 [3], the USEPA mandated that utilities must eliminate CSO events. As a result more than 700 cities began planning the implementation of solutions to CSO events.

Typical solutions to mitigate CSOs include: the separation of sanitary and storm water collection systems, transformation of impermeable surfaces into permeable surfaces through green infrastructure, expansion of treatment plants, and construction of large reservoirs or tunnels to temporary store excess flows. All of these solutions are extremely expensive and highly disruptive to the community.

Water collection systems present significant challenges that make the problem of eliminating CSOs difficult. Water collection systems consist of thousands of nodes connected by hundreds of miles of pipes that are buried out of sight. Its performance is difficult to measure and rarely monitored. In addition, water moves in sewer systems mainly by gravity with flows governed by highly non-linear equations such as the 1-D Saint Venant equation. Other factors that affect its operation and dynamics are surface permeability, ambient temperature, moisture, and wind, frequency of street sweeping, time of the year, among others. Also, travel times throughout the sewer system can be significant. For this city, water can take up to 24 hours to travel from the network's edge to the treatment facility. This means that the optimal control requires as an input rainfall forecast which is best treated as a stochastic process.

This paper describes a CPS called CSOnet, a wireless (RF) network of microprocessors controlling sensing and actuation devices within a municipal sewer system. The objective is to control wastewater flows in real-time in order to maximize the total amount of water that is transported to the wastewater treatment plant by the network, maximize storage and conveyance capacity of the sewer network during storm events and thereby minimizing the overflowed volume. This control is subject to constraints on link capacity within the network as well as safety constraints to minimize flooding.

\section{ARCHITECTURE}

The presented solution attempts to take advantage of existing conveyance and storage capacity of the collection system as storm events present high temporal and spatial variability literally leaving pipes half empty while others are 
overflowing. By doing so, CSOnet is able to redirect flows from areas that are overwhelmed to areas with lesser hydraulic loads. This solution however requires real time monitoring and control of flows within the collection system during the storm event.

CSOnet was designed as a dense wireless sensor actuator network that could reliably and economically monitor the collection system and implement a distributed control system. CSOnet consists of three main components:

\subsection{Monitoring Hardware}

This component is composed by the actual hardware installed in the sewer and that is in charge of acquiring and processing sensor data, transmitting the data, and, in some cases, adjusting the position of a valve, gate, or movable weir through an electrical actuator. There exists three types of devices: a sensor node deployed in the sewer which is in charge of measuring hydraulic conditions, a gateway which is typically mounted above ground and is in charge of collecting information from the sensor nodes and using a Wide Area Network (WAN) connection to retransmit such data to a database, and repeaters which fill in the spatial gaps when sensor nodes are distant from a gateway.

The sensor node was designed to withstand the harsh conditions encountered in sewer systems. Corrosive and explosive gases, constant humidity, biofilm formation, submerged conditions, large debris traveling at $20 \mathrm{ft} / \mathrm{sec}$ or more are common in sewer systems. Furthermore, since sewer lines are usually installed under city roads, maintenance requires traffic control in potentially dangerous conditions.

The sensor node is based on the original Mica2 mote [4]. The new sensor node, called the Chasqui mote, is outfitted with industrial hardened inputs for up to four analog signals and one digital RS232 input. Each input has its own separate power control mechanism where sensor power can be entirely removed. An 8Mbyte non-volatile flash memory provides storage for sensor data. A highly accurate Real Time Clock (RTC) chip allows precise time tracking with low temperature drifting. A lithium battery and a series of switching power supplies form the power subsystem of the Chasqui mote. The microprocessor is able to independently remove power from other subsystems to conserve power. The Chasqui board draws from the $13 \mathrm{~V}$ lithium battery currents up to $1 \mathrm{~A}$ during data transmission but ramps down to less than $30 \mathrm{uA}$ during its sleep mode. While the sensor nodes can accommodate a virtually unlimited number of batteries, a battery pack consisting of 4 D-sized Lithium batteries in series provides about 12 months of uninterrupted operation when sampling and transmitting at 5 minute intervals. Among the improvements over the original Mica2 board, the device was outfitted with a spread spectrum radio operating at $900 \mathrm{MHz}$.

Later, with the reductions in cost of cellular data, the device was provided with a way to instead use a cellular modem for data transmission. The use of cellular telemetry at the sensor node level provides with an inexpensive way of sending data directly to the system's database. However, cellular systems take a significantly longer amount of time to register with the carrier's network. As a result, to achieve the same 12 months of battery life the $900 \mathrm{MHz}$ system is able to achieve, the sensor node must transmit its data only every 3 hours. On each transmission the sensor node is able to transmit all its data which continues to be sampled every
5 minutes. An internal programmable threshold places the sensor node in "alert mode" during abnormal conditions. In "alert mode" the cellular sensor node transmits every 5 minutes. Because this mode can rapidly deplete the battery, it is used sparingly.

Each sensor node consists of two parts: a telemetry part and a sensor part. The telemetry part is located at street level and is built under a composite manhole cover to allow for surface level radio transmissions. An explosion-proof box (to comply with deployment of electronics in an explosive environment) houses the Chasqui node and its battery pack. The antenna located on the outside of the explosion proof box is a slot antenna designed to broadcast radio frequency signals out of the manhole. The sensor part is typically located in the pipe and consists of third party available level or flow sensors.

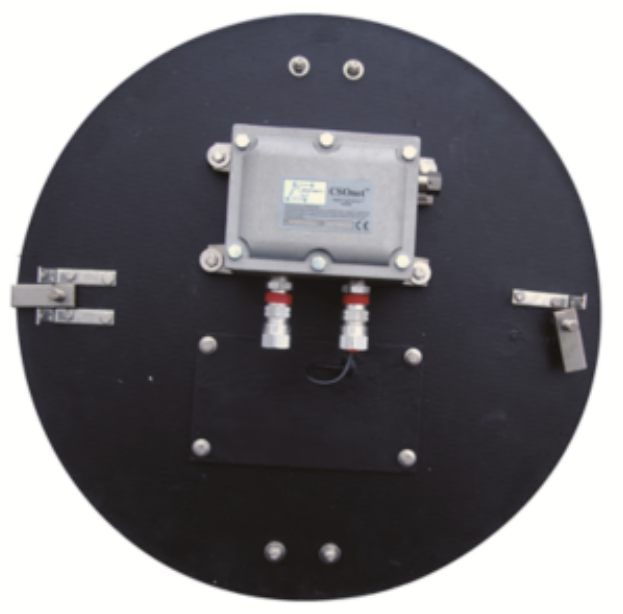

Figure 1: Underneath of a sensor node. Visible are the composite manhole cover, the explosion proof box containing all the electronics, a cam-lock system to maintain the lightweight cover in place, and the antenna (under the explosion proof box).

The sensors used by CSOnet are a combination of level sensors and ultrasonic area velocity flow meters. Sensor data is typically sampled at 5 minutes intervals.

The gateway consists of an embedded PC running a Linuxbased operating system, a $900 \mathrm{MHz}$ radio to communicate with the sensor nodes, and a WAN connection (typically a cellular modem). The gateway is usually permanently powered through a connection to the electrical grid or through a solar cell system. The gateway also contains interface systems to control sewer infrastructure. For example 4 to 20 mA outputs or RS232 Modbus protocols are used to open and close gates or to set pump stations to a speed setpoint.

The repeater consists of a similar architecture to a sensor node, however, since the repeater is typically installed above ground, the repeater does not require a cover, sensors, or an explosion proof box. Repeaters are either battery powered or permanently powered.

\subsection{Communication Subsystem}

CSOnet was initially designed to exclusively use its $900 \mathrm{MHz}$ radio to transmit data by establishing an ad-hoc, multi-hop wireless network. In this scheme, data from various sensors 
is aggregated to a gateway device with Wide Area Network (WAN) connectivity. The permanently powered gateway allows the sensor network data to be accessible at all times. To conserve power, the gateway performs a synchronization function where its role is to synchronize all the network to sleep and be awake at the same time to allow for multi-hop interconnectivity. To do this, the gateway synchronizes its own internal clock to a National Institute of Standards and Technology (NIST) time server through the WAN connection. Then it broadcasts a synchronization beacon daily to all devices on its network. Each node synchronizes its RTC chip to the time provided by the synchronization beacon. The need for an accurate RTC chip stems from the fact that there can be a drastic difference in temperature between the sensor nodes installed in the sewer (typically around 12C) and gateway and repeater nodes that are at street level (exposed to temperatures that can be as low as $-20 \mathrm{C}$ on the winter and as high as $40 \mathrm{C}$ on the summer).

Devices use a stateless routing protocol where each device wakes up at 5 minute intervals and transmits data to a neighboring node. The routing protocol named Stateless Gradient-Based Persistent Routing establishes routes from the source to the destination based on a gradient structure imposed to the network. Each node in the network has a gradient number that is an indication of how close the node is to the destination gateway.

A gateway initiates the generation of the gradient number by sending out a beacon message. As the beacon message travels outward from the gateway point, nodes receiving the beacon generate their gradient based on the number of hops traveled by the beacon and their previous gradient number. The beacon message is transmitted using traditional flooding techniques.

When a node in the network desires to transmit a data message it appends its own gradient information corresponding to the destination and the destination ID. The message is then sent to all neighbors. The message will be forwarded only by those neighboring nodes with lower gradient number than the transmitter. In this way, messages travel âĂIJdown-gradientâĂİ towards the data sink. This method resembles the so called Directed Diffusion algorithm [5]. In order to increase reliability, anonymous acknowledgment messages are used for each forwarded message. If a forwarding node does not receive an acknowledgment that its message was heard by a lower gradient node, it will try to retransmit.

Since there is no explicit routing information generated, the computational complexity of the protocol is minimum as opposed to traditional Bellman-Ford or Dijkstras based approaches. Moreover, the network is inherently resilient to node failure as long as network remains connected with some link success probability. All data exchange happen in a period of approximately 3 seconds.

The 5 minute sampling time is designed to capture hydraulic transients in the sewer system. While transients with a time constant of less than 5 minutes do occur, they are rare. Hydraulic modeling software is typically used to design the system. Several years' worth of rainfall are simulated in the modeling software to identify events that require faster sampling times.

As previously mentioned, the advent of machine-to-machine applications has significantly reduced cellular hardware costs and data rates making it possible to have a cellular commu-

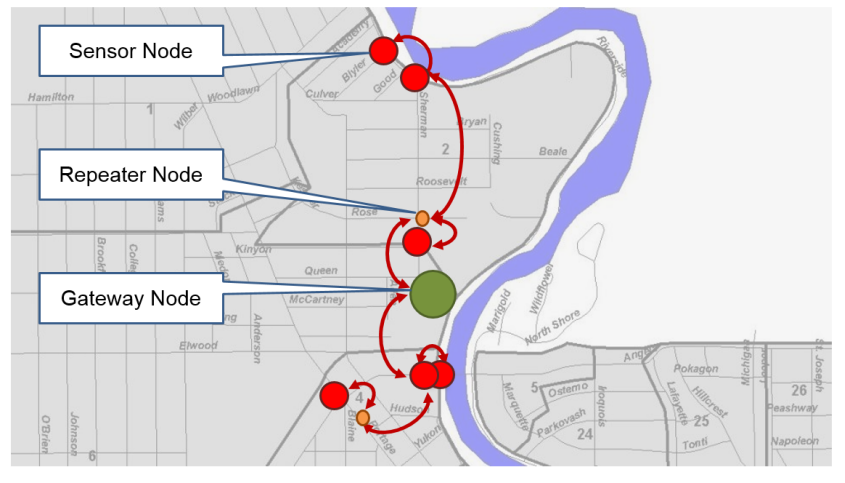

Figure 2: Example of a deployed network. The red circles represent the sensor nodes, the orange circles represent repeaters, the green circle represents a gateway.

nication system at the sensor node level. This effectively eliminates the need for gateways and repeaters. However, due to power constrains, 5 minute sampling and transmission has to be limited to extreme circumstances. This communication methodology is usually reserved for sites where data for control is not needed but where system operation can be analyzed for maintenance purposes.

\subsection{Distributed Control Subsystem}

The control of the collection system is performed by dynamically controlling actuators throughout the collection system. Actuator typically utilized in collection systems are movable weirs, valves, gates, and pump stations that are electrically controlled. Some of these actuators where already be part of the existing infrastructure (e.g. pump stations). Other actuators had to be installed as part of the implementation of CSOnet (e.g. gates). By changing the set points on these actuators, CSOnet is able to actively reconfigure the sewer system to better accommodate the time-varying hydraulic loads in the sewer system. As a storm moves through the city, these actuators mirror the precipitation patterns by balancing the hydraulic loads of different parts of the sewer network.

Given the scale of the collection system, the unreliability of actuator systems and communication systems (especially during storm events), all control algorithms were required to have a distributed architecture. Each actuator-enabled device is able to directly communicate with devices upstream and downstream.

Several control schemes have been implemented. A problem identified on the early stages of implementation was that the City's main interceptor line was not balanced. The interceptor line is a sewer pipe that collects water from different sewersheds and transports it to the wastewater treatment plant. On its original design, each sewershed was assigned a maximum allowed flow to enter the interceptor. Any excess flows are redirected to the river through a CSO diversion structure. The CSO structure consists of a narrow throttle line that allows normal dry weather flow to go from the sewershed to the interceptor and a weir that overflows when the throttle line capacity is exceeded.

It was noted that since it rarely rains evenly throughout the city, some CSO structures were overflowing while others are not. This meant that the interceptor line was not at full 
capacity throughout the storm event.

To solve the problem, a supplemental dynamically controlled throttle line was added (see Figure 3). The control of the throttle line is performed through the use of an electrically actuated pinch valve which allows more water to enter the interceptor when the sensor network detected available capacity downstream from the valve. The valve needs to close if capacity is exceeded to avoid surcharging and street flooding from occurring.

A control scheme based in Pontryagin's maximum principle which produces a switching algorithm is used to balance flows through an interceptor sewer line by searching where the hydraulic constraint and then actively managing it [7]. The control strategy is implemented on gateway nodes located where the throttle valves are installed. The gateway nodes are able to communicate with each other through the use of a peer-to-peer communication system implemented at the WAN level.

Throughout the life of the system, other control algorithms have been implemented. For example, a control schemes that mimics a free market economy of devices competing for downstream capacity produces a PID-like control.

Recent advances in weather forecasting have allowed the implementation of probabilistic control systems that predicts the amount of runoff that will enter the sewer system and creates capacity to accommodate the additional flows. For example, the city has storm water detention basins which have a dual use of detaining storm water prior to entering the sewers and providing sustaining aquatic life (as a recreational water body or pond). Because must be able to sustain aquatic life, the pond must be maintained at a minimum level. However, in order to effectively function as a detention basin, the pond must make room for receiving storm water from the surrounding areas. The control system receives precipitation forecasts that present the probability distribution function of future rainfall. The control system then is able to determine, with a specified level of risk based on the probability distribution functions, how much water must it drain from the pond in preparation for the storm such that retains its minimum level while minimizing the risk of contributing to overflows at the sewer system.

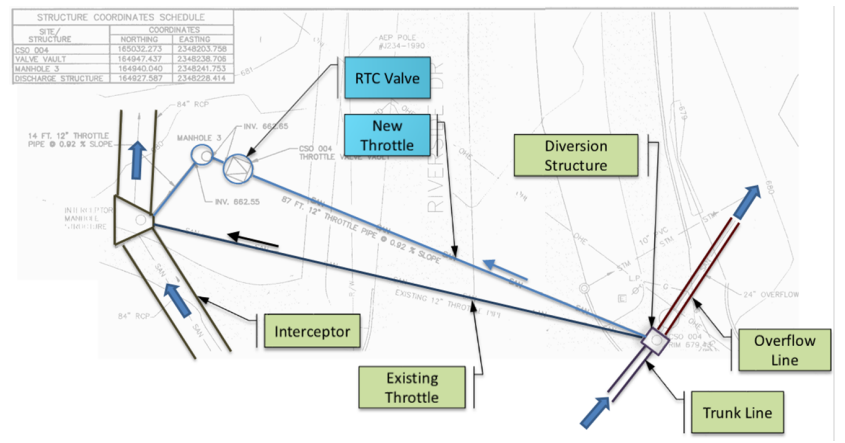

Figure 3: Sewer infrastructure for balancing flow in the interceptor. The blue lines (new throttle line and RTC valve) where added as part of this project.

\section{IMPLEMENTATION}

The CSOnet monitoring system was installed in 2008 and its control subsystem was implemented in 2010. Currently, the CSOnet system consists of 150 wireless nodes monitoring 111 locations. These locations include 36 discharge locations, 28 sewer interceptor points, 42 trunk line points, and 5 basins. The system is divided in 32 subnets interconnected through the use of a cellular data network. The CSOnet system controls 9 valves and 3 movable weirs.

The communication system works well with overall throughputs exceeding $85 \%$ at all times. Communication path patterns are constantly changing as during the summer months tree vegetation forces messages that traverse along streets while during winter, lower hop counts are observed traversing across blocks. However, during the winter time, snow and slush increase the number of retries for messages initiated by sensor nodes embedded on the road.

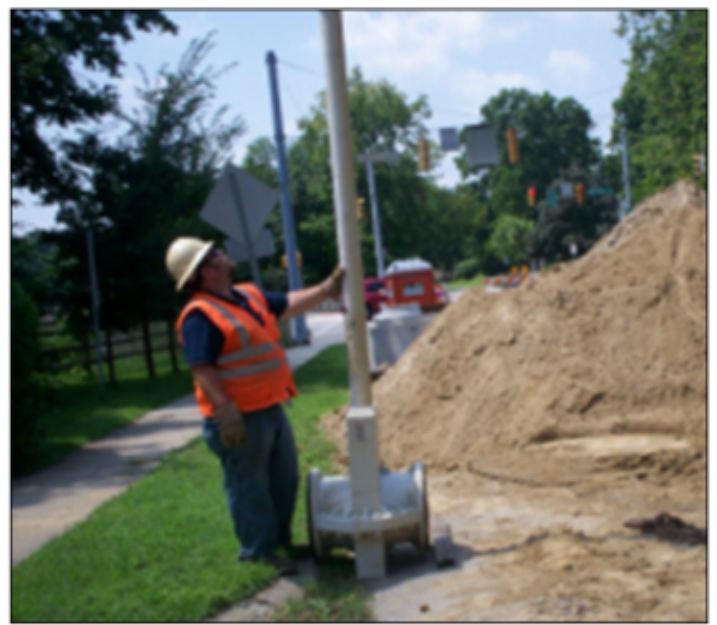

Figure 4: Installation of a valve for an auxiliary throttle valve.

The implementation of the control algorithm has been done in stages to gain the trust of the utility staff. The initial state of the implementation of the control algorithm was done by verifying the effectiveness of the system via hydraulic simulations. On the field, increasing levels of control aggressiveness were achieved by limiting the actuator dynamical range.

The system has been effective at reducing overflows not only by dynamically controlling the valves and movable weirs but by allowing the utility to increase the hydraulic performance of the collection system through better maintenance. For example, the sensor network is able to detect areas that operate outside of the normal regime observed historically. Sewer cleaning crews have been to troubleshoot the issue and routinely pull large amounts of gravel or other debris that otherwise limit the conveyance capacity of the sewer system.

\section{RESULTS}

Computer simulations showed a $25 \%$ reduction of overflows using synthetic rainfall based on precipitation measured in 1995 (called "typical year" precipitation for regulatory purposes). Actual measurements indicate a more drastic reduction of overflows, although it is worth noting that other improvements to the sewer system have been done over the same period. 


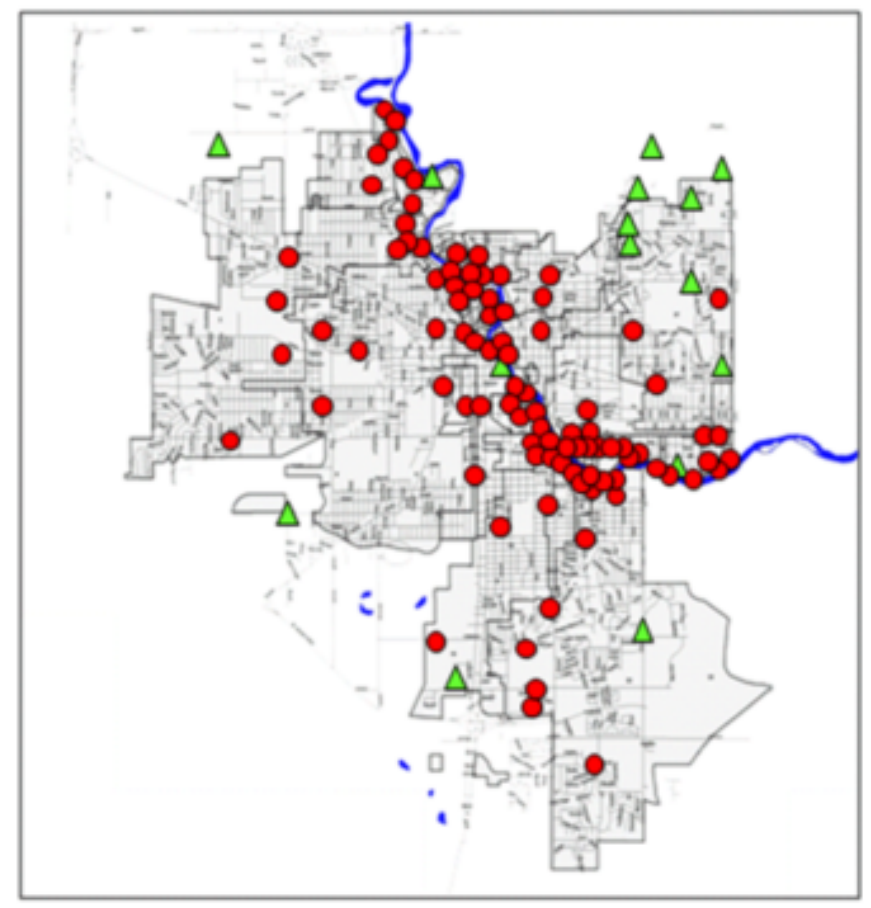

Figure 5: CSOnet sensor network deployment. The red circles represent the sensor node locations. The green triangles represent the location of other telemetry systems under the utility control.

Table 1 shows a performance index that relates the total yearly CSO volume to the total yearly amount of precipitation. Before CSOnet was commissioned (2006-2008) the performance index hovered around $41 \mathrm{Mgal} / \mathrm{in}$. After CSOnet was fully installed the CSO volume decreased by more than $50 \%$ per inch of precipitation.

Figure 6 shows the performance of the system during 2014. The top chart shows the overflow $(1,187 \mathrm{Mgal})$ and underflow (flows sent to the treatment facility, 3,3036Mgal) as simulated by the hydraulic model of the original system prior to CSOnet and using 2014 rain data. The middle chart shows the overflows (458MGal) and underflows (3,236MGal) that actually occurred as measured by the sensor network (post CSOnet implementation). The bottom chart shows the overflow volumes prevented by the CSOnet system from

Table 1: CSOnet performance

\begin{tabular}{|cccc|}
\hline Year & $\begin{array}{c}\text { Total } \\
\text { Precipitation } \\
\text { (in) }\end{array}$ & $\begin{array}{c}\text { Total } \\
\text { Overflow } \\
\text { (Mgal) }\end{array}$ & $\begin{array}{c}\text { Performance } \\
\text { Index } \\
\text { (Mgal/in) }\end{array}$ \\
\hline 2006 & 45.0 & 2111 & 46.9 \\
2007 & 42.0 & 1432 & 34.1 \\
2008 & 48.0 & 2054 & 42.8 \\
\hline 2009 & 36.5 & 1199 & 32.8 \\
2010 & 29.5 & 804 & 27.3 \\
\hline 2011 & 41.9 & 890 & 21.2 \\
2012 & 29.4 & 445 & 15.1 \\
2013 & 35.8 & 715 & 20.0 \\
2014 & 39.9 & 458 & 11.5 \\
\hline
\end{tabular}

overflowing as measured by the sensor network (312MGal).

Original Collection System Performance (2014 rain)

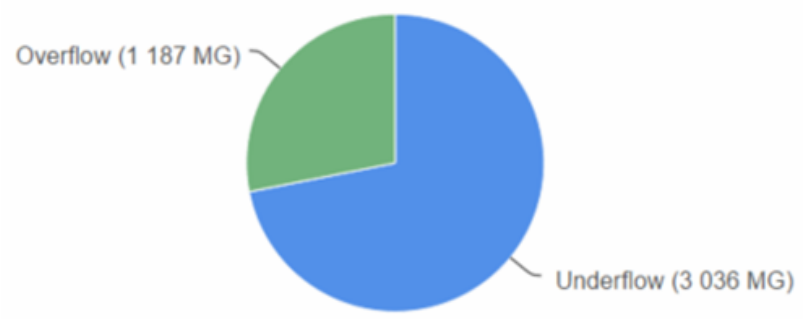

\section{Collection System Performance with CSOnet}

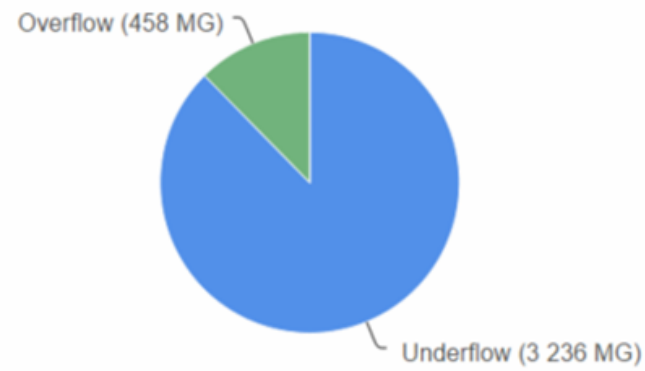

Overflows Prevented by CSOnet (312.02MGal)
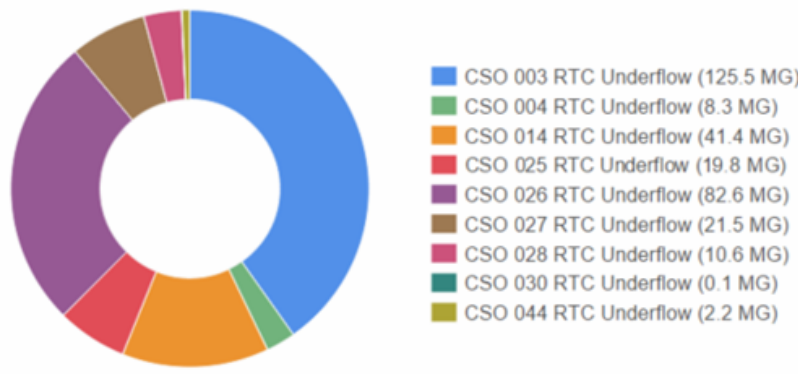

Figure 6: System performance during 2014.

It is worth noting that mass balances discrepancies exist between the hydraulic model of the original system, the current CSOnet-augmented system, and the overflow reductions assigned to CSOnet. The original system shows a total water volume of $4,223 \mathrm{Mgal}$ while the current system shows a total of 3,694Mgal (a 12\% discrepancy). This discrepancy can be attributed mainly to the removal of stormwater inputs to the system (sewer separation) as well as model tolerances. A full attribution analysis is currently underway.

\section{CONCLUSIONS AND FUTURE WORK}

The CSOnet system has successfully mitigated CSO events over the last 4 years and demonstrated the impact of CPS systems on a smart city environment. The impact of the CPS system has been the reduction of the cost of original CSO mitigation plan by approximately $\$ 150$ Million.

Furthermore, recent analysis of the sewer system indicated that more opportunities exist to further reduce overflows. An entire new continuous interceptor line running across the network can be formed through three small connections 
between previously unconnected nodes. However, legal and compliance related challenges remain as utility regulators are confronted with new technology that was not conceived when plans were made to mitigate CSOs.

Future work on CSOnet will concentrate in exploring the more systematic ways to implement control systems based, perhaps on graph theory and agent based computing to more effectively synthesize control algorithms.

Also, much remains to be explored on how the CSOnet infrastructure can be leveraged to further improve the level of service of other urban infrastructure. Currently, CSOnet's infrastructure is being leveraged to test other applications such as optimization of drinking water facilities, optimization of salt application during winter time, and monitoring of water quality in river streams.

\section{REFERENCES}

[1] U. S. E. P. Agency. Combined sewer overflow control policy. Federal Register, 59(75):18688-18698, 1994.

[2] U. S. E. P. Agency. Report to congress: Implementation and enforcement of cso control policy. EPA, 833-R-01-003, 2001.

[3] U. S. Congress. Federal water pollution control act. 33 U.S.C. SS1251-1387, 1972.

[4] J. Hill. A software architecture supporting networked sensors - Master's thesis. U.C. Berkeley, Department of Electrical Eng. and Computer Science, Berkeley, CA, 2000.

[5] M. Maroti. Directed food-routing framework. Technical Report ISIS-04-502, Institute for Software Integrated Systems, Vanderbilt University, 2004.

[6] L. Montestruque and M. Lemmon. Csonet: a metropolitan scale wireless sensor-actuator network. International Workshop on Mobile Device and Urban Sensing (MODUS), 2008.

[7] P. Wan and M. Lemmon. Distributed flow control using embedded sensor-actuator networks for the reduction of combined sewer overflow events. Proceedings of the IEEE Conference on Decision and Control, 2007. 